\title{
Practice and Exploration of Artificial Intelligence Education in Universities of Political Science and Law with Python
}

\author{
Yang Liu ${ }^{1, a,{ }^{*}}$ and Jincheng Huang ${ }^{2, b}$ \\ 1Department of Information Science and Technology, East China of Political Science and Law, \\ No.555 Longyuan Road, Songjiang District, Shanghai, 201620, China \\ 2Department of Information Engineering, Yancheng Institute of Technology, Hope Avenue Road 1, \\ Yancheng City, Jiangsu Province, 224051, China \\ alyang@ecupl.edu.cn, bHuangjc@ycit.cn \\ ${ }^{*}$ Corresponding author
}

\begin{abstract}
Keywords: Al Education, Universities of Political Science and Law, Practice and Exploration, Python, Non-Computer Science Major.
\end{abstract}

\begin{abstract}
In the context of artificial intelligence, legal education is also facing a huge impact. For students of political and law colleges and universities, through the process of learning of Python programming, on the one hand, the ability of students to solve problems can be cultivated. On the other hand, students can use modern methods to solve problems in their professional field, and finally understand and master the top technological dynamics of the world. This paper summarizes and analyzes the practice and exploration of Python programming courses in the past four years. Practice shows that Python-based artificial intelligence courses are more conducive to non-computer majors. Meanwhile, the role of teachers should be changed from knowledge transferers to guides to help students improve their ability in the process of knowledge acquisition.
\end{abstract}

\section{Introduction}

With the widespread use and integration of information technology in all fields of society, especially the popularity of the Internet, and the implementation of strategic planning goals such as "big data", "cloud computing" and "artificial intelligence" in the world, human society has entered the "wisdom era"[1]. Artificial intelligence has emerged in various fields and achieved remarkable achievements. At present, innovation has become the main driving force for economic and social development, and knowledge innovation is the core element of national competitiveness. These are inseparable from the combination of innovative and innovative talents. With the rapid development and continuous application of artificial intelligence, big data and other technologies, as well as the rapid changes of human society in the future, the goal of university education should not only focus on the infusion of knowledge content, but also focus on excellent self-learning ability and rapid adaptation. The cultivation of abilities and data literacy creates talents with solid foundation, broad knowledge, strong ability, high quality and innovative spirit[2].

In the process of talent cultivation in universities, there are a large amount of data analysis in different professions and research directions. If we can combine the analysis and processing of judicial documents such as judicial documents and judicial cases with computer technology, and promote the deep integration of artificial intelligence technology and justice, we will make full use of big data technology to serve judicial practice such as assisted judgment[3]. Teachers need to encourage students of different majors and students with different specialties to collaborate in the after-school homework and course design process to achieve cross-integration, give full play to their own characteristics and advantages, teach students in accordance with their aptitude, and let students learn and apply their skills, enjoy the satisfaction of learning[4].

According to Google's trend analysis, since January 2019, Python has surpassed Java and become the top language. First of all, this is closely related to the third wave of artificial intelligence, Python is known as "the only way to artificial intelligence." Secondly, compared with $\mathrm{C} / \mathrm{C}++$ and JAVA, 
Python language is simple, clear and easy to use[5]. Finally, Python has a strong community and ecological environment, with a rich third-party library, without the need to manually write code one line at a time from the bottom, and can directly call the packaged function to achieve "building block" programming.

\section{Artificial intelligence Course based on Python}

\subsection{The necessity of general education in artificial intelligence courses}

Professional artificial intelligence education mainly cultivates professional and technical talents in the field of artificial intelligence. It is difficult to balance the expansion and integration with other related disciplines while deepening the basic knowledge of the profession. That is, students trained in professional artificial intelligence education usually focus only on the technical level itself rather than the human needs. The third wave of artificial intelligence is due to its remarkable achievements in the professional field[6,7].

The introduction of artificial intelligence courses in political and law colleges will promote the intersection and integration of judicial disciplines with big data and artificial intelligence disciplines, which will help to cultivate composite talents with interdisciplinary background and promote artificial intelligence in judicial data processing. The application is very important.

\subsection{Features of Python and the courses}

Python is a language that represents simple ideas, which is very easy to use, write and learn cause of its extremely simple grammar[8]. Programs written in the Python language do not need to be compiled into binary code, as opposed to compiler languages such as $\mathrm{C} / \mathrm{C}++$. Inside the computer, the Python interpreter converts the source code into an intermediate form of byte code, and then translates it into a machine language used by the computer and runs it, which makes it very portable. Compared to other major languages such as $\mathrm{C}++$ and Java, Python implements object-oriented programming in a very powerful and simple way. In addition, Python has great scalability and a rich standard library.

The Python Language Programming course of East China University of Political Science and Law mainly guides students to learn, weaken grammar rules, highlights field applications, and emphasizes big data analysis, allowing students to feel the charm of artificial intelligence. At the same time, an interest group was established after class to train excellent cases, and actively lead students to participate in various practical innovation activities, which stimulated students' interest in learning and subjective initiative; cultivated students' self-learning ability, planning ability, and ability to analyze problems and solve problems; It takes advantage of the students' spare time to provide support for the development of composite innovative talents that meet the requirements of the times and the intersection of "law" and "computer".

\subsection{Research paradigm}

The widespread use of the Internet and information technology and the depth of big data research, artificial intelligence, and big data technology have changed the way scientific research. For political and law colleges, data-based analysis and research has brought about a new situation in disciplinary research, which will promote the transformation of research paradigms.

The initial research paradigm is to first define the rules, regulate the social phenomena through rules, and then feedback the comments from the social phenomena to modify the rules. The definition of rules is often complex and abstract, and modern society develops rapidly. When new rules are formulated and applied in practice through feedback, social imagination may have evolved again as Fig.1 shown below. 


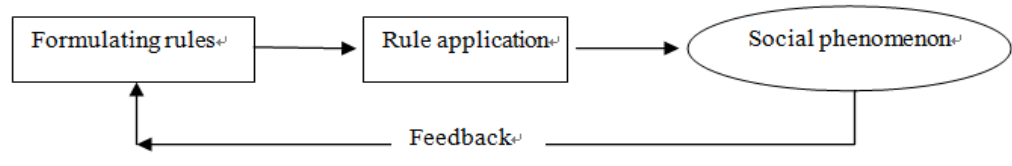

Fig. 1. Traditional research paradigm

The research of big data of law based on artificial intelligence is the latest research direction of empirical law, which will bring about the paradigm revolution of legal research. Different from previous research paradigms, legal empirical research is to analyze the data of social imagination and draw the current rules. The data is different and the laws are different. Therefore, rules can be formulated and revised quickly and accurately, and social phenomena can be predicted. The cultivation of undergraduate students in colleges and universities should be stratified, part of which enters the society, and some select graduate students to continue their studies. No matter which kind of students are concerned, it is necessary and beneficial to understand the world's cutting-edge research trends and research methods.

\section{Course teaching case}

\subsection{Course content}

The artificial intelligence course for non-computer major students actually involves two core issues, one is how to teach and the other is what to teach. For students in specialized fields such as computers, the task of artificial intelligence courses is to enable students to understand and master the various core algorithms and applications of artificial intelligence, and to cultivate solid research and application talents. For non-related majors, especially most students in political and law colleges, it is not easy to complete simple programming. Let them understand what is the neural network re-code implementation, the difficulty will be great, after all, the industry has a specialization. Therefore, the main content of this part of the artificial intelligence course includes: the development of artificial intelligence, the application of artificial intelligence and Python using modular programming. You can directly build a neural network and use it without having to master the underlying technology.

\subsection{Course cases}

In the course of practice, the teaching content of the course mainly includes two parts, basic knowledge and application cases. After several rounds of course practice and curriculum system construction, we have abandoned the previous method of focusing on basic knowledge and the disconnection between theory and practice. Instead, we adopted the case appreciation practice as the main line, the basic knowledge as the secondary line, and the follow-up teaching mode. Because the course is aimed at students of all majors in the school, the goal of these students to learn programming is not to become a programmer, but a learning activity and process of "learning through programming" to achieve logical thinking, data literacy, computational thinking, The cultivation of comprehensive skills such as hands-on practice has made practice and exploration for the interdisciplinary, new liberal arts, and even the construction of new law.

Therefore, in the teaching practice arrangement, from the macro level, with the case as a guide, lead the students to gradually study. Whether it is textbook construction or classroom teaching, this idea is reflected. First, give a basic case, let students have an intuitive feeling, and then gradually deepen to the basic knowledge needed for case realization, and integrate relatively boring theory.

\subsubsection{Legal analysis}

The case is given 10 judicial documents, requiring students to count the laws used in them and the number of times each law is used. Among the elective students, many students with grammar backgrounds learn Python with their professional domain data to achieve cross-disciplinary integration. After class, the number of paperwork continued to increase, from 10 to 100,000. Using the computing resources of the big data teaching platform to realize the "calculation" of big data, let students feel the charm of "computation" and actually feel the impact of technology on work. As 
shown in Figure 2 below. The figure analyzes 20,000 judicial judgments and analyzes the use of the law. The first result is in the 20,000 documents, the Civil Procedure Law of the People's Republic of China, the first one hundred and forty-five The article used 887 times, and the 253th used 760 times.

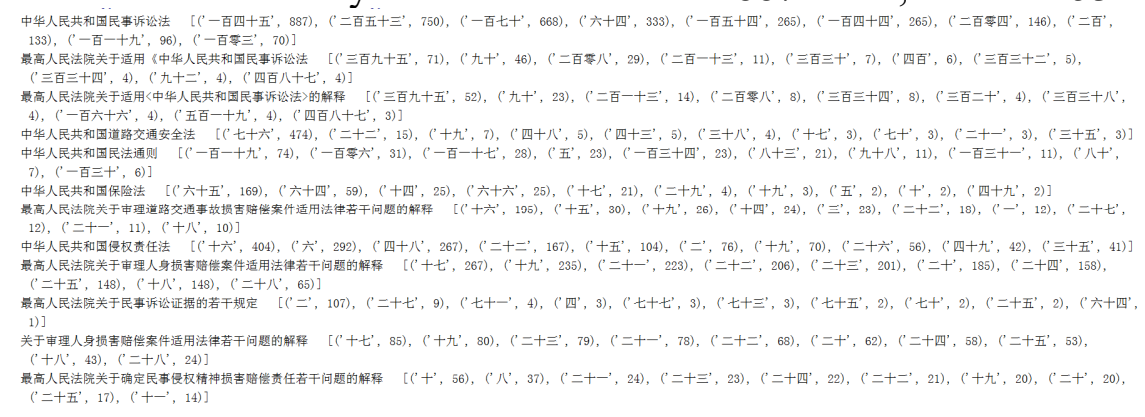

Fig. 2. Legal statistics

\subsubsection{Natural language computability}

In this case, the corpus is trained using the word embedding technique and the Chinese word segmentation technique, and the Chinese vocabulary is mapped to an independent space, and "embedded" into a vector. After the vector, the word can be calculated. As shown in Figure 3 below. Among them, the corpus used in the model 1 model is 5000 news, and the corpus used in the model 2 model is 20,000 judicial theft documents. From the results of word training, the same words, such as "theft", are different in different corpora, so the more professional the training corpus, the better the effect. The vocabulary is expected to be the closest to the given word in the expected library. It can be seen from the results that in the field of judicial documents, because of the different styles of writing, the meaning of "theft" is also expressed, and the words used by each person are different. Subsequent deepening of the case also included the extraction of case elements and the push of similar cases and the automatic writing of judicial documents.
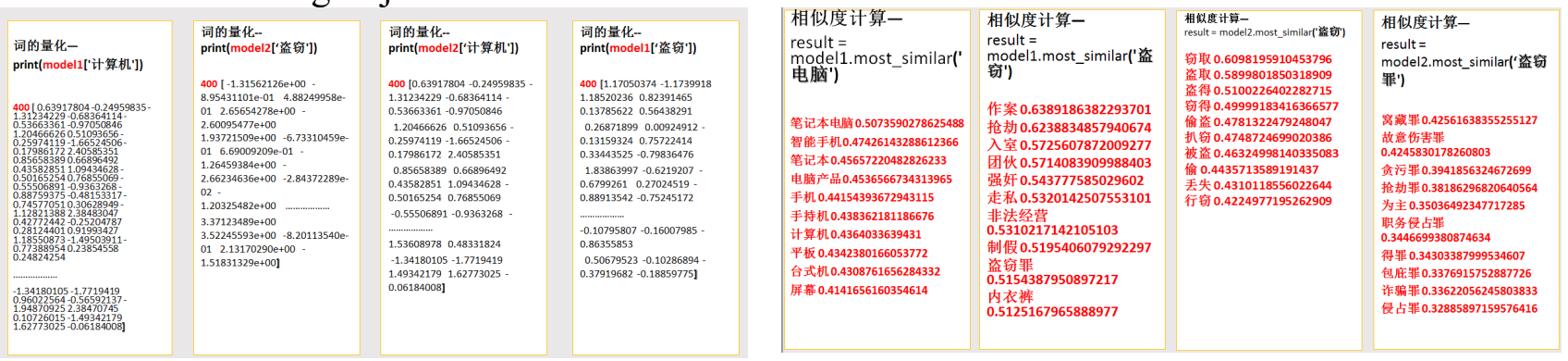

Fig. 3. Word embedding and the similarity calculation

\section{Summary}

Based on the general Python course opened by the author at East China University of Political Science and Law, this paper expounds the necessity of Python artificial intelligence courses, the characteristics of Python and the characteristics of Python courses, and the role in the transformation of research paradigm. Combining with the author's own teaching practice, through the in-depth interpretation of the practice and exploration links of teaching materials construction, course teaching and application cases, the East China University of Political Science and Law is taken as an example to analyze and explore the artificial intelligence education of political and law colleges based on Python curriculum. As a result of practice and exploration, while promoting the improvement of students' comprehensive ability and strengthening the cultivation of compound talents, the research results can provide useful reference and reference for other Chinese political and legal colleges to open relevant courses. 


\section{Acknowledgment}

This article is the Shanghai Higher Education Society project "Artificial Intelligence Interdisciplinary Curriculum Design and Organization for Law Talents Training" (GJEL183) , 2019Shanghai Municipal Education Commission undergraduate key curriculum construction project(No.228) ,the Ministry of Education's first batch of industry-university cooperation collaborative education project "Arts and universities artificial intelligence curriculum construction seminar training One of the phased results of (201801154067) and 2019 East China University of Political Science and Law first-class undergraduate construction leading plan of the second classroom construction project.

\section{References}

[1] X. Kui, W. Liu, J. Xia and H. Du, Research on the improvement of python language programming course teaching methods based on visualization, 2017 12th International Conference on Computer Science and Education (ICCSE), Houston, TX, pp. 639-644, 2017.

[2] C. Sotomayor-Beltran, G. W. Z. Segura and A. Roman-Gonzalez, Why should Python be a compulsory introductory programming course in Lima (Peru) universities, 2018 IEEE International Conference on Automation/XXIII Congress of the Chilean Association of Automatic Control (ICA-ACCA), Concepcion, pp. 1-4, 2018.

[3] T. R. Fernandes, L. R. Fernandes, T. R. Ricciardi, L. F. Ugarte and M. C. de Almeida, Python Programming Language for Power System Analysis Education and Research, 2018 IEEE PES Transmission \& Distribution Conference and Exhibition - Latin America (T\&D-LA), Lima, pp. $1-5,2018$.

[4] Y. Song, Redesigning a Computer Science Capstone Course with Micro-credentials, 2018 IEEE Frontiers in Education Conference (FIE), San Jose, CA, USA, pp. 1-5, 2018.

[5] C. Cabo, Effectiveness of Flowcharting as a Scaffolding Tool to Learn Python, 2018 IEEE Frontiers in Education Conference (FIE), San Jose, CA, USA, pp. 1-7, 2018.

[6] R. Lyu, Y. Kuo and C. Liu, Machine Translation of English Identifiers in Python Programs into Traditional Chinese, 2016 International Computer Symposium (ICS), Chiayi, pp. 622-625, 2016.

[7] R. Fraanje, T. Koreneef, A. Le Mair and S. de Jong, Python in robotics and mechatronics education, 2016 11th France-Japan \& 9th Europe-Asia Congress on Mechatronics (MECATRONICS) /17th International Conference on Research and Education in Mechatronics (REM), Compiegne, pp. 014-019, 2016.

[8] G. Zhao, S. Zhao, C. Zou and Z. Wang, Exploration of teaching method of Python Programming based on the case of technical problem, 2017 12th International Conference on Computer Science and Education (ICCSE), Houston, TX, pp. 600-603, 2017. 